PROCEEDINGS OF THE

AMERICAN MATHEMATICAL SOCIETY

Volume 32, Number 2, April 1972

\title{
MINIMAL PRESENTATIONS FOR CERTAIN METABELIAN GROUPS
}

\author{
D. G. SEARBY AND J. W. WAMSLEY
}

\begin{abstract}
Let $G$ be a finite $p$-group, $d(G)=\operatorname{dim} H^{1}(G, Z \mid p Z)$ and $r(G)=\operatorname{dim} H^{2}(G, Z \mid p Z)$. Then $d(G)$ is the minimal number of generators of $G$, and we say that $G$ is a member of a class $\mathscr{G}_{p}$ of finite $p$-groups if $G$ has a presentation with $d(G)$ generators and $r(G)$ relations. The main result is that any outer extension of a finite cyclic $p$-group by a finite abelian $p$-group belongs to $\mathscr{G}_{p}$.
\end{abstract}

1. Introduction. Let $G$ be a finite $p$-group. We have

$$
\begin{aligned}
& d(G)=\operatorname{dim} H^{1}(G, Z / p Z)=\operatorname{dim}_{Z_{p}}\left(G / G^{\prime} G^{p}\right), \\
& r(G)=\operatorname{dim} H^{2}(G, Z / p Z),
\end{aligned}
$$

$d(G)$ being the minimal number of generators of $G$. If there is a presentation

$$
G=F / R=\left\{x_{1}, \cdots, x_{n} \mid R_{1}, \cdots, R_{m}\right\}
$$

where $F$ is the free group on $x_{1}, \cdots, x_{n} ; n=d(G)$, and $R$ is the normal closure in $F$ of $R_{1}, \cdots, R_{m}$, we have always $m \geqq r(G)=d\left(R /[F, R] R^{p}\right)$ (see, for example [2]). We say that $G$ belongs to a class $\mathscr{G}_{p}$ of finite $p$ groups if there is such a presentation with $n=d(G)$ and $m=r(G)$. Such a presentation is said to be minimal.

$G$ is said to be an extension of a group $K$ by $H$ if $H$ is a normal subgroup of $G$, and $G / H \cong K$. $G$ is said to be an outer extension of $K$ by $H$ if $G$ is an extension of $K$ by $H$, and $d(G)=d(K)+d(H)$.

In this paper it is shown that if $K$ is a finite cyclic $p$-group, and $H$ is a finite abelian $p$-group, then any outer extension of $K$ by $H$ belongs to $\mathscr{G}_{p}$. The case $n=1$ has been covered in [2].

\section{Basic lemmas.}

LEMMA 1. Let $G$ be a finite p-group with presentation $G=F / R$ where $d(G)=d(F)$, and let $d\left(R /[F, R] R^{p}\right)=m$. If $R_{1}, \cdots, R_{m}$ is any set of $m$ elements of $R$, linearly independent in $R$ modulo $[F, R] R^{p}$, and $K=F / S$ where $S$ is the normal closure of $R_{1}, \cdots, R_{m}$ in $F$; then $G$ is the maximal

Received by the editors January 25, 1971.

AMS 1970 subject classifications. Primary 20D15, $20 \mathrm{~F} 05$.

Key words and phrases. Presentation, outer extension, finite cyclic p-group, finite abelian $p$-group, Frattini subgroup.

(c) American Mathematical Society 1972 
p-factor group of $K$ in the sense that if $A$ is any finite p-group which is a factor group of $K$, then $A$ is a factor group of $G$.

Proof. Let $\Gamma_{k}(F)$ be the $k$ th term of the lower central series of $F$. Any $p$-factor group of $K=F / S$ with class $k$ and exponent $q=p^{\alpha}$ will necessarily be a factor group of

$$
K /\left(\Gamma_{k}(F) F^{q}\right) \cong(F / S) /\left(\Gamma_{k}(F) F^{q} S / S\right) \cong F /\left(\Gamma_{k}(F) F^{q} S\right) .
$$

Thus it will suffice to show that

$$
R \subseteq \Gamma_{k}(F) F^{q} S
$$

since if so then $F /\left(\Gamma_{k}(F) F^{q} S\right)$ is a factor group of $F / R=G$, and any $p$ factor of $F / S$ will hence be a factor of $G$.

Let $U=[F, R]$ and let $T$ be the normal closure of $R^{q}$ in $F$; then $S T U=R$ and $U=[R, F]=[S T U, F] \subseteq[U, F] S T \subseteq[U, F, F] S T \subseteq \cdots$ so that $U \subseteq$ $\Gamma_{k}(F) S T$ for all $k$. Now $T \subseteq F^{q}$ so that $U S T=R \subseteq \Gamma_{k}(F) F^{q} S$ which establishes (1), and hence the lemma.

Corollary. Let $N=\left\{x_{1}, \cdots, x_{n} \mid R_{i_{1}}, \cdots, R_{i_{t}}\right\}$ where $R_{i_{1}}, \cdots, R_{i_{t}}$ is any subset of $R_{1}, \cdots, R_{m}$. If $N$ is a finite p-group, then $G \in \mathscr{G}_{p}$.

Proof. Let $H=\left\{x_{1}, \cdots, x_{n} \mid R_{1}, \cdots, R_{m}\right\}$, then $H$ is a factor of $N$, sc $H$ is a finite $p$-group, and by the lemma, $H=G$.

LEMMA 2. Let $G=F / R=\left\{x_{1}, \cdots, x_{n} \mid R_{1}, \cdots, R_{m}\right\}$ and $G / N=$ $\left\{x_{1}, \cdots, x_{n} \mid R_{1}, \cdots, R_{m}, S_{1}, \cdots, S_{t}\right\}=F / S$. Then if $R_{i_{1}}, \cdots, R_{i_{s}}$ are linearly independent in $S$ modulo $[F, S] S^{p}$, they are linearly independent in $R$ modulo $[F, R] R^{p}$.

Proof. The natural mapping of $R /\left([F, R] R^{p}\right)$ into $S /\left([F, S] S^{p}\right)$ is clearly a homomorphism, and hence a linear transformation of the respective vector spaces.

THEOREM 1. Let $K=\left\{x_{1}, \cdots, x_{n} \mid R_{1}, \cdots, R_{m}\right\}$ be a finite p-group, then $G=\left\{x_{1}, \cdots, x_{n} \mid R_{1}, \cdots, R_{m}, S_{1}, \cdots, S_{t}\right\}$ belongs to $\mathscr{G}_{p}$ if

$$
H=\left\{x_{1}, \cdots, x_{n} \mid R_{1}, \cdots, R_{m}, S_{1}, \cdots, S_{t}, T_{1}, \cdots, T_{n}\right\}
$$

has a minimal presentation $H=\left\{x_{1}, \cdots, x_{n} \mid R_{1}, \cdots, R_{m}, U_{1}, \cdots, U_{v}\right\}$ for suitable $U_{i}$.

Proof. By Lemma $2, R_{1}, \cdots, R_{m}$ are linearly independent, and by Lemma 1 and the Corollary, $G \in \mathscr{G}_{p}$.

The following well-known theorem, which is stated without proof, is due to D. Epstein [1].

THEOREM 2. If $G$ is a finite abelian p-group with $d(G)=n$, then $G$ has a minimal presentation with $n$ generators and $\frac{1}{2} n(n+1)$ relations. 
Let $A$ be a finite abelian $p$-group generated by $\left\{a_{1}, \cdots, a_{n}\right\}$, and let $G=\left\{a_{1}, \cdots, a_{n}, x \mid R_{1}, \cdots, R_{m}\right\}$ be any outer extension of a finite cyclic $p$-group by $A$. Then if $\phi(G)$ denotes the Frattini subgroup of $G$, since the extension is outer, $\phi(G) \cap A=\phi(A)$. If amongst the defining relations of $G$ there occurs

$$
x a_{i} x^{-1}=a_{1}^{\alpha_{i 1}} \cdots a_{i}^{\alpha_{i i}} \cdots a_{n}^{\alpha_{i n}}
$$

i.e. $x a_{i} x^{-1} a_{i}^{-1}=a_{1}^{\alpha_{i 1}} \cdots a_{i}^{\alpha_{i i}-1} \cdots a_{n}^{\alpha_{i n}}$, then since $x a_{i} x^{-1} a_{i}^{-1} \in \phi(G)$,

$$
a_{1}^{\alpha_{i 1}} \cdots a_{i}^{\alpha_{i i-1}} \cdots a_{n}^{\alpha_{i n}} \in \phi(A)=A^{p},
$$

and thus $\alpha_{i j} \equiv 0(\bmod p)$ if $i \neq j$, and $\alpha_{i i} \equiv 1(\bmod p)$.

LEMMA 3. Let

$$
\begin{aligned}
G=\left\{a_{1}, \cdots, a_{n}, x \mid a_{i}^{m_{i}}, x^{-k} a_{1}^{\lambda_{1}} \cdots\right. & a_{n}^{\lambda_{n}}, x a_{i}^{-1} x^{-1} a_{1}^{\alpha_{i 1}} \cdots a_{n}^{\alpha_{i n}} \\
& \left.(i=1, \cdots, n) ;\left[a_{i}, a_{j}\right](i>j)\right\},
\end{aligned}
$$

where $m_{i}=p^{\dot{\beta}_{i}}, k=p^{\delta}, \lambda_{i}=k_{i} p^{\delta_{i}}, k_{i} \neq \equiv 0(\bmod p), \alpha_{i j} \equiv 0(\bmod p)$ if $i \neq j$, $\alpha_{i i} \equiv 1(\bmod p)$, be an outer extension of a finite cyclic $p$-group by a finite abelian p-group, for which $\left\{a_{1}, \cdots, a_{n}, x\right\}$ is a minimal generating set. Then $G$ has a presentation

$$
\begin{aligned}
& G=\left\{b_{1}, \cdots,\right. b_{n}, x \mid b_{i}^{m_{i}} w_{i}(p)(i<n), b_{n}^{m_{n}}, x^{-k} b_{1}^{\pi_{1}}, \\
& x b_{i}^{-1} x^{-1} b_{1}^{v_{i 1}} \cdots b_{i}^{v_{i i}} b_{i+1}^{\pi_{i+1}}(i<n), x b_{n}^{-1} x^{-1} b^{v_{n 1}} \cdots b_{n}^{v_{n n}}, \\
& {\left.\left[b_{i}, b_{j}\right](i>j,(i, j) \neq(n, 1)), b_{1}^{-1} b_{n}^{-1} b_{1}^{m} b_{n}\right\} }
\end{aligned}
$$

where $m_{i}=p^{\beta_{i}}, w_{i}(p) \in\left\langle b_{i+1}^{p}, \cdots, b_{n}^{p}\right\rangle, k=p^{\delta}, \pi_{1}=p^{\delta_{1}}, \pi_{i}=p^{v_{i}}(i>1),\left\{v_{i j}\right\}$ is some set of integers satisfying $v_{i j} \equiv 0(\bmod p)$ if $i \neq j, v_{i i} \equiv 1(\bmod p)$, and $m=1+\lambda p^{\mu}$, where $\lambda$ is an integer, and $p^{\mu}$ is the exponent of $G$.

Proof. We may suppose $\delta_{1} \leqq \delta_{i}$ for all $i$; set $\lambda_{1}^{\prime}=k_{i} p^{\delta_{i}-\delta_{1}}$ and $\pi_{1}=p^{\delta_{1}}$. Then $x^{k}=a_{1}^{\lambda_{1}} \cdots a_{n}^{\lambda_{n}}=\left(a_{1}^{\lambda_{1}{ }^{\prime}} \cdots a_{n}^{\lambda^{\prime}}\right)^{\pi_{1}}=b_{1}^{\pi_{1}}$. As $\lambda_{1}^{\prime} \not \equiv 0(\bmod p),\left\{b_{1}, a_{2}, \cdots\right.$, $\left.a_{n}, x\right\}$ is a generating set, $b_{1}^{m_{1}} \in\left\langle a_{2}^{p}, \cdots, a_{n}^{p}\right\rangle$ and $\left[b_{1}, a_{i}\right]=1$ for all $i$.

Now, let $i<n$, and suppose the required changes have been made for all $j \leqq i$. Then

$$
x b_{i} x^{-1}=b_{1}^{v_{i 1}} \cdots b_{i}^{v_{i i}}\left(a_{i+1}^{z_{i+1}^{\prime}} \cdots a_{n}^{\alpha_{i n}^{\prime}}\right)=b_{1}^{v_{i 1}} \cdots b_{i}^{v_{i i}}\left(a_{i+1}^{\alpha_{i+1}^{\prime \prime}} \cdots a_{n}^{\alpha_{i n}^{\prime \prime}}\right)^{\pi_{i+1}}
$$

as in the first step, where $\alpha_{i+1}^{\prime \prime} \not \equiv 0(\bmod p), \pi_{i+1}=p^{v_{i+1}}$. Let $b_{i+1}$ be the term inside the brackets. Then $\left\{b_{1}, \cdots, b_{i+1}, a_{i+2}, \cdots, a_{n}, x\right\}$ is a generating set, $b_{i+1}^{m_{i+1}} \in\left\langle a_{i+2}^{p}, \cdots, a_{n}^{p}\right\rangle$ (if $i<n-1$, otherwise $b_{n}^{m_{n}}=1$ ), $x b_{i} x^{-1}=$ $b_{1}^{v_{i 1}} \cdots b_{i}^{v_{i}} b_{i+1}^{\pi_{i+1}},\left[b_{i+1}, a_{j}\right]=1$ for all $j$, and all the congruences on the $\left\{v_{i j}\right\}$ hold, since the change of generators does not change the Frattini subgroup, and the remarks immediately preceding this lemma still apply. 
Thus by induction we construct $b_{1}, \cdots, b_{n}$ satisfying the required relations. The process terminates at $b_{n}$, and we still have $x b_{n} x^{-1}=$ $b_{1}^{v_{n 1}} \cdots b_{n}^{v_{n n}}$. At this step we may go through the argument again, replacing each occurrence of $\left\langle a_{j}^{p}, \cdots, a_{n}^{p}\right\rangle$ by $\left\langle b_{j}^{p}, \cdots, b_{n}^{p}\right\rangle$. Clearly the order of

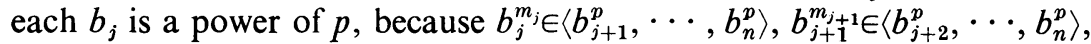
-. etc., and $b_{n}^{m_{n}}=1$, each $m_{i}$ being a power of $p$. Also, if the exponent of $G$ is $p^{\mu}$, we may replace the defining relation $\left[b_{n}, b_{1}\right]=1$ by $b_{n} b_{1}=b_{1}^{m} b_{n}$, where $m=1+\lambda p^{\mu}$ for some positive integer $\lambda$. This completes the proof.

Note. In the above proof, $v_{i j}$ may be replaced by $v_{i j}+s p^{\mu}$ for some integer $s$, and for all $i$ and $j$.

LEMMA 4. Let $A(t), B(t)$ and $C(t)$ be rational polynomials in $t, \mu a$ fixed nonzero integer, and $K$ and $L$ infinite sets of integers. Then it is possible to choose integers $\kappa \in K$ and $\lambda \in L$ such that the polynomials $A(t)$ and $D(t)=$ $\kappa B(t)+\lambda C(t)+\mu$ are coprime.

Proof. Let $A(t)$ be factorized over the rationals into irreducible factors $A_{1}(t), \cdots, A_{r}(t)$. For each $i, 1 \leqq i \leqq r$, there are four possibilities:

(i) $A_{i}(t) \mid B(t)$ and $A_{i}(t) \mid C(t)$-then $A_{i}(t) \nmid D(t)$ for all $\kappa$ and $\lambda$.

(ii) $A_{i}(t) \mid B(t)$ and $A_{i}(t) \nmid C(t)$-then there is at most one $\lambda$ such that $A_{i}(t) \mid D(t)$, since if $\lambda_{1}$ and $\lambda_{2}$ have this property:

$$
A_{i}(t) \mid \kappa_{1} B(t)+\lambda_{1} C(t)+\mu \text { and } A_{i}(t) \mid \kappa_{2} B(t)+\lambda_{2} C(t)+\mu,
$$

hence $A_{i}(t) \mid\left(\kappa_{1}-\kappa_{2}\right) B(t)+\left(\lambda_{1}-\lambda_{2}\right) C(t)$ which is impossible unless $\lambda_{1}=\lambda_{2}$.

(iii) $A_{i}(t) \nmid B(t)$ and $A_{i}(t) \mid C(t)$-then there is at most one $\kappa$ such that $A_{i}(t) \mid D(t)$ - the proof is as for (ii).

(iv) $A_{i}(t) \nmid B(t)$ and $A_{i}(t) \nmid C(t)$-then for each $\kappa \in K$, there is at most one $\lambda \in L$ for which $A_{i}(t) \nmid D(t)$ and conversely, since if, for $\kappa \in K$ and $\lambda_{1}$ and $\lambda_{2} \in L$,

$$
A_{i}(t) \mid \kappa B(t)+\lambda_{1} C(t)+\mu \text { and } A_{i}(t) \mid \kappa B(t)+\lambda_{2} C(t)+\mu,
$$

then $A_{i}(t) \mid\left(\lambda_{1}-\lambda_{2}\right) C(t)$, which is impossible unless $\lambda_{1}=\lambda_{2}$. Similarly for the converse.

Now, define $K_{1} \subset K$ by $\kappa \in K_{1}$ iff for some $i$, case (iii) applies, and $\kappa \in K$ is the unique integer permitted by the argument, and define $L_{1} \subset L$ similarly. As $K_{1}$ and $L_{1}$ are finite, $K^{\prime}=K-K_{1}$ and $L^{\prime}=L-L_{1}$ are infinite, and clearly if $\kappa \in K^{\prime}$ and $\lambda \in L^{\prime}, A_{i}(t) \nmid D(t)$ if (i), (ii) or (iii) applies. Choose any $\kappa \in K^{\prime}$ and define $L_{2} \subset L^{\prime}$ by $\lambda \in L_{2}$ iff for some $i$, case (iv) applies and $\lambda$ is the unique second member of the pair $(\kappa, \lambda)$ permitted by the argument. Then $L_{2}$ is finite, so $L^{\prime \prime}=L^{\prime}-L_{2}$ is infinite, and by the construction, if $\kappa \in K^{\prime}$, $\lambda \in L^{\prime \prime}$, then

$$
A_{i}(t) \nmid \kappa B(t)+\lambda C(t)+\mu \text { for each } i=1, \cdots, r .
$$

Hence $A(t)$ and $\kappa B(t)+\lambda C(t)+\mu$ are coprime. 
LeMmA 5. Let $p, q_{1}, \cdots, q_{r}$ be distinct primes, then it is possible to find an integer $k$ such that, for $n>0$,

$$
\left(1+k p^{\alpha}\right)^{n}-1 \not \equiv 0\left(\bmod q_{1}, \cdots, q_{r}\right) .
$$

Proof. $\quad p^{\alpha}$ is prime to $q_{1} \cdots q_{r}$ so by the division algorithm there exists an integer $k$ such that $k p^{\alpha} \equiv-1\left(\bmod q_{1} \cdots q_{r}\right)$. Then

so

$$
\left(1+k p^{\alpha}\right)^{n}-1 \equiv-1\left(\bmod q_{1} \cdots q_{r}\right)
$$

$$
\left(1+k p^{\alpha}\right)^{n}-1 \equiv-1\left(\bmod q_{1}, \cdots, q_{r}\right) .
$$

\section{The main theorem.}

THEOREM 3. Let

$$
\begin{array}{r}
G=\left\{a_{1}, \cdots, a_{n}, x \mid a_{i}^{m_{i}}, x^{-k} a_{1}^{\lambda_{1}} \cdots a_{n}^{\lambda_{n}}, x a_{i}^{-1} x^{-1} a_{1}^{\alpha_{i 1}} \cdots a_{n}^{\alpha_{i n}}\right. \\
\left.(i=1, \cdots, n) ;\left[a_{i}, a_{j}\right](i>j)\right\}
\end{array}
$$

where $m_{i}=p^{\beta i}, k=p^{\delta}, \lambda_{i}=k_{i} p^{\delta_{i}}, k_{i} \not \equiv 0(\bmod p), \alpha_{i j} \equiv 0(\bmod p)$ if $i \neq j$, $\alpha_{i i} \equiv 1(\bmod p)$, be any outer extension of a finite cyclic p-group by a finite abelian p-group for which $d(G)=n+1$. Then $G \in \mathscr{G}_{p}$.

Proof. By Lemma 3, $G$ has a presentation

$$
\begin{array}{r}
G=\left\{b_{1}, \cdots, b_{n}, x \mid x^{-k} b_{1}^{\pi_{1}} ; x b_{i}^{-1} x^{-1} b_{1}^{v_{i 1}} \cdots b_{i}^{v_{i i}} b_{i+1}^{\pi_{i+1}}(i<n),\right. \\
x b_{n}^{-1} x^{-1} b_{1}^{v_{n 1}} \cdots b_{n}^{v_{n n}} ;\left[b_{i}, b_{j}\right](i>j,(i, j) \neq(n, 1)), \\
\left.b_{1}^{-1} b_{n}^{-1} b_{1}^{m} b_{n} ; b_{i}^{m_{i}} w_{i}(p)(i<n), b_{n}^{m_{n}}\right\}
\end{array}
$$

where $k=p^{\delta}, \pi_{1}=p^{\delta_{1}}, \pi_{i}=p^{v_{i}}(i>1), v_{i j} \equiv 0(\bmod p)$ if $i \neq j, v_{i i} \equiv 1(\bmod p)$, $m=1+\lambda p^{\mu}, m_{i}=p^{\beta_{i}}, w_{i}(p) \in\left\langle b_{i+1}^{p}, \cdots, b_{n}^{p}\right\rangle$. We abbreviate this presentation to

$$
G=\left\{b_{1}, \cdots, b_{n}, x \mid R_{1}, \cdots, R_{t}, b_{i}^{m_{i}} w_{i}(p)(i<n), b_{n}^{m_{n}}\right\} .
$$

With this notation we define

and

$$
K=\left\{b_{1}, \cdots, b_{n}, x \mid R_{1}, \cdots, R_{t}\right\}
$$

$$
\begin{array}{r}
H=\left\{b_{1}, \cdots, b_{n}, x \mid R_{1}, \cdots, R_{t}, b_{i}^{m_{i}} w_{i}(p)(i<n), b_{n}^{m_{n}} ;\right. \\
\left.b_{j}^{p}(j=1, \cdots, n)\right\} .
\end{array}
$$

Now $H$ is an elementary abelian group, and by Theorem 2 , has a minimal presentation with $\frac{1}{2}(n+1)(n+2)$ relations-but $t=1+n+C_{2}^{n}=\frac{1}{2}(n+1) \times$ $(n+2)-n$, so $H$ has a minimal presentation

$$
H=\left\{b_{1}, \cdots, b_{n}, x \mid R_{1}, \cdots, R_{t}, b_{j}^{p}(j=1, \cdots, n)\right\},
$$

and $H \in \mathscr{G}_{p}$. Thus $R_{1}, \cdots, R_{t}$ are linearly independent, and to apply 
Theorem 1 and thereby prove the theorem, it remains only to show that for a suitable choice of $v_{i j}$ and $\lambda, H$ is a $p$-group.

We have $x^{k}=b_{1}^{\pi_{1}}$ so $x b_{1}^{\pi_{1}} x^{-1}=b_{1}^{\pi_{1}}$, which implies $b_{1}^{\left(v_{11}-1\right) \pi_{1}} b_{2}^{\pi_{2} \pi_{1}}=1$,

$$
x b_{2}^{\pi_{2} \pi_{1}} x^{-1}=x b_{1}^{\left(1-v_{11}\right) \pi_{1}} x^{-1}=b_{1}^{\left(1-v_{11}\right) \pi_{1}}=b_{2}^{\pi_{2} \pi_{1}},
$$

which implies $b_{1}^{v_{21} \pi_{2} \pi_{1}} b_{2}^{\left(v_{22}-1\right) \pi_{2} \pi_{1}} b_{3}^{\pi_{3} \pi_{2} \pi_{1}}=1$. We continue as in the last step for $b_{3}, \cdots, b_{n-2}$, obtaining $x b_{n-2}^{\pi_{n}-{ }^{2} \cdots \pi_{1}} x^{-1}=b_{n-2}^{\pi_{n}-2}{ }^{\cdots \pi_{1}}$ which implies

$$
b_{1}^{v_{n-2} \pi_{n-2} \cdots \pi_{1}} \cdots b_{n-2}^{\left(v_{n-2 n-2-1}\right) \pi_{n-2} \cdots \pi_{1}} b_{n-1}^{\pi_{n-1} \cdots \pi_{1}}=1 .
$$

For $b_{n-1}$ we recall that $b_{n} b_{1}=b_{1}^{m} b_{n}$ applies, and we derive

$$
b_{1}^{v_{n-11} S(m)} b_{2}^{v_{n-1} 2^{\pi_{n-1} \cdots \pi_{1}} \cdots b_{n-1}^{\left(v_{n-1} n-1-1\right) \pi_{n-1} \cdots \pi_{1}}} b_{n}^{\pi_{n} \cdots \pi_{1}}=1 .
$$

From this and the preceding equations, $b_{n}^{\pi_{n} \cdots \pi_{1}} \in g p\left\{b_{1}\right\}$, so $b_{n}^{\pi_{n} \cdots \pi_{1}{ }^{2}} \in g p\left\{b_{1}^{\pi_{1}}\right\}=$ $g p\left\{x^{k}\right\}$ so that $x b_{n}^{\pi_{n} \cdots \pi_{1}{ }^{2}} x^{-1}=b_{n}^{\pi_{n} \cdots \pi_{1}{ }^{2}}$, and

$$
b_{1}^{v_{n 1} T(m)} b_{2}^{v_{n 2} \pi_{n} \cdots \pi_{1}{ }^{2}} \cdots b_{n}^{\left(v_{n n-1) \pi_{n} \cdots \pi_{1}{ }^{2}}=1\right.}
$$

where in (i) and (ii), $S(m)$ and $T(m)$ are polynomials in $m$, which are independent of $v_{n n}, v_{n-11}$ and $v_{n 1}$. From (i) and (ii) and earlier derivations, we may derive

$$
b_{n}^{\pi_{n} \cdots \pi_{1}}=b_{1}^{-c_{1}-v_{n-11} S(m)}, \quad b_{n}^{\left(v_{n n}-1\right) \pi_{n} \cdots \pi_{1}{ }^{2}}=b_{1}^{-c_{2}-v_{n 1} T(m)}
$$

where $c_{1}$ and $c_{2}$ are nonzero integers independent of $v_{n n}$. Thus

$$
b_{n}^{\left(v_{n n}-1\right) \pi_{n} \cdots \pi_{1}{ }^{2}}=b_{1}^{-\left(v_{n n}-1\right) \pi_{1} v_{n-1} S(m)-\left(v_{n n}-1\right) \pi_{1} c_{1}}=b_{1}^{-c_{2}-v_{n 1} T(m)} .
$$

By suitable choice of $v_{n n}$ we may ensure that

$$
c_{3}=\left(v_{n n}-1\right) \pi_{1} c_{1}-c_{2} \neq 0
$$

then $b_{1}^{\psi(m)}=1$, where $\psi(m)=\left(v_{n n}-1\right) \pi_{1} v_{n-1} S(m)-v_{n 1} T(m)+c_{3}$.

Also, from (i): $b_{n} b_{1} b_{n}^{-1}=b_{1}^{m}$, so $b_{n}^{\sigma} b_{1} b_{n}^{-\sigma}=b_{1}^{m^{\sigma}}$. If we put $\sigma=p^{\pi_{n} \cdots \pi_{1}}$, then $b_{n}^{\sigma}$ is a power of $b_{1}$, so $b_{n}^{\sigma} b_{1} b_{n}^{-\sigma}=b_{1}$, and we have

$$
b_{1}^{m^{\sigma}-1}=1, \quad b_{1}^{\psi(m)}=1,
$$

so that $\left|b_{1}\right|$ is the greatest common divisor of $m^{\sigma}-1$ and $\psi(m)$. Now $S(m)$ and $T(m)$ are independent of $v_{n-11}$ and $v_{n 1}$, so that by Lemma 4 we can choose these coefficients so that the polynomials have no common factor containing $m$.

Now if two polynomials are coprime in this sense, the Euclidean algorithm shows that it is possible to find a linear combination of them which is an integer, say $q_{1}^{t_{1}} \cdots q_{k}^{t_{k}} p^{t_{0}}$-but if $\left|b_{1}\right|$ divides $m^{\sigma}-1$ and $\psi(m)$, then it must divide this number, whence, since $m=1+\lambda p^{\mu}$, by Lemma 5 it is possible to choose $\lambda$ such that $m^{\sigma}-1$ is prime to $q_{1}, \cdots, q_{k}$. From 
this we deduce that $\left|b_{1}\right|$ is a power of $p$, and thus that the order of every generator is $p$-power.

Thus $K$ is a finite $p$-group, and by the earlier remarks, this is sufficient to complete the proof.

\section{REFERENCES}

1. D. Epstein, Finite presentations of groups and 3-manifolds, Quart. J. Math. Oxford Ser. (2) 12 (1961), 205-212. MR 26 \#1867.

2. J.-P. Serre, Cohomologie galoisienne, 3rd ed., Lecture Notes in Math., no. 5, Springer-Verlag, Berlin and New York, 1965. MR 34 \#1328.

3. J. W. Wamsley, The deficiency of metacyclic groups, Proc. Amer. Math. Soc. 24 (1970), 724-726. MR 41 \#3576.

School of Mathematics, The Flinders University of South Australia, Bedford Park, South Australia 